Original Research Paper

\title{
Mining, Isolation and Identification of Siderophore Synthesis Gene from Brevibacillus brevis GZDF3
}

\author{
${ }^{1,2}$ Miaomiao Sheng, ${ }^{2}$ Huake Jia, ${ }^{2}$ Xiaomai Tao, ${ }^{2}$ Lina Zeng, \\ ${ }^{2}$ Tingting Zhang, ${ }^{3,4}$ Zuquan Hu, ${ }^{1,3,4}$ Zhu Zeng and ${ }^{2,3} *$ Hongmei Liu \\ ${ }^{1}$ Basic Medical Sciences, Guizhou Medical University, \\ Guiyang 550025, People's Republic of China \\ ${ }^{2}$ Medical Biotechnology Engineering Research Center, \\ Guizhou Medical University, Guiyang 550025, People's Republic of China \\ ${ }^{3}$ Key Laboratory of biological and medical engineering, \\ Guizhou Medical University, Guiyang 550025, People's Republic of China \\ ${ }^{4}$ Guizhou Immunology Cell and Antibody Engineering Research Center, \\ Guizhou Medical University, Guiyang 550025, People's Republic of China
}

\section{Article history}

Received: 09-07-2018

Revised: 08-08-2018

Accepted: 27-08-2018

Corresponding Author:

Hongmei Liu

Medical Biotechnology

Engineering Research Center,

Guizhou Medical University,

Guiyang 550025, People's

Republic of China and

Key Laboratory of biological and medical engineering,

Guizhou Medical University,

Guiyang 550025, People's

Republic of China

Email:283599737@qq.com

\begin{abstract}
Objective of this paper is to excavate the siderophore synthesis gene from Brevibacillus brevis GZDF3 and verify its type and antibacterial effects. The method is using genome mining technology to analyze the siderophore synthesis genes and the phylogenetic tree of each synthesis gene was constructed separately. Iron free medium was utilized to induce the synthesis of siderophore and CAS liquid detection method was used for qualitative and quantitative analysis on siderophore. The type of siderophore was preliminaries identified by Arnow and its antibacterial effects were analyzed according to the agar punching method. The results show that a siderophore synthesis gene cluster with $83 \%$ similarity to Petrobactin was found in Brevibacillus brevis GZDF3 genome. Iron free medium could induce siderophore synthesis and the optimal incubation time cultured in iron free medium was $30 \mathrm{~h}$ and $48 \mathrm{~h}$. Antagonistic strain GZDF3 had the capacity to synthesize catechol-type siderophore. Also, GZDF3 had a powerful antibacterial effect on pathogenic fungus Fusarium oxysporum of rotted root on Pinellia ternata. Therefore, Brevibacillus brevis GZDF3 can produce catechol-type siderophore in an iron-deficient culture medium, which was also a main antifungal active substance.
\end{abstract}

Keywords: Brevibacillus brevis, Genome Mining, Petrobactin Siderophore, CAS Detection, Antibacterial

\section{Introduction}

Iron is one of the essential trace elements of all living organisms and it participates in many biological metabolism, such as photosynthesis, respiration, oxygen transport, gene regulation and DNA biosynthesis. Iron is an abundant metal, being the fourth most plentiful element in the Earth's crust. The majority of iron in the environment is in the form of insoluble ferric oxide/hydroxide complexes and to limit the growth of pathogens in vivo, serum contains ironbinding proteins such as transferrin and lactoferrin that maintain a very low concentration of free iron $\left(\sim 10^{-18}\right.$ M). In order to obtain the iron necessary for survival, microbes have evolved many ways to obtain iron and synthetic siderophore is one of the important approaches (Carroll et al., 2017; Serrano, 2017).

The siderophores are low molecular weight, high affinity iron chelators that are secreted in response to the iron limitation to scavenge iron and their collection system is the most diverse and broadly distributed iron uptake mechanism for microorganisms (Zawadzka et al., 2009). The molecular weight is between 500-1500 Da and the siderophore specific transporter systems import the Fe-siderophore across the cell membrane to the cytoplasm (Fukushima et al., 2013). Indeed, there are more than 500 known species of siderophores and siderophores known species of siderophores and siderophores have been classified according to the $\mathrm{Fe}(\mathrm{III})$-coordinating groups: Catecholate, hydroxamaces, 
carboxylates or mixed (Arora and Verma, 2017; Barry and Challis, 2009). At present, siderophore also can be classified by their mode of mode biosynthesis, Non-Ribosomal Peptide Synthetase (NRPS)-dependent or NRPS-independent biosynthesis (NIS) (Han et al., 2013). The synthesis mechanism of NRPS has been studied deeply, such as Pyoverdine synthesized by Pseudomonas aeruginosa, the synthesis of Enterbactin by Enterobacteriaceae and more. Although there are limited studies on NIS, there is a definite understanding of its synthesis mechanism, such as Aerobactin synthesized by Enterobacteriaceae, Staphyloferrin synthesized in Staphylococcus aureus, Desferrioxamine in the Streptomyces and more. However, recent studies have found that there is a siderophores with NRPS and NIS in nature, such as Petrobactin, which is synthesized by Bacillus anthraci (Hagan et al., 2016). Bacillus anthraci siderophore Petrobactin is encoded by as $b A B C D E F$ genes, of which AsbAB belong to the NIS family and the AsbCDE synthetases belong to the NRPS family and a dehydrase (AsbF) (Lee et al., 2007).

Studies have shown that under the environment of iron deficiency, Rhizobium leguminosarum (Storey et al., 2006) and Bacillus megaterium can both synthesize Schizokinen. However, Paenibacillus Ash strains (Hertlein et al., 2014), Bacillus subtilis (Grossman et al., 1993) and Bacillus cereus (Hayrapetyan et al., 2016) can synthesize catecholates Bacillibactin. Bacillus anthracis and Bacillus cereus can not only synthesize Bacillibactin, but also synthesize Petrobactin. The complete Bacillibactin and Petrobactin synthesis gene cluster have been found in the genome of Bacillus thuringiensis (Wilson et al., 2006; Hollensteiner et al., 2016). Bacterial siderophore is not only a wide variety of species, but also a variety of functions. The siderophores in biocontrol bacteria have strong antagonistic effects on a variety of pathogenic fungus. In China, the study of Chen et al. (2006) showed that the Pseudomonas sp. WCS358r strain had a potent inhibitory effect on the growth of chestnut germs and the germination of conidia, while the deletion mutant JM218 of siderophore had almost no inhibition effect. After adding $200 \mu \mathrm{mol} / \mathrm{L} \mathrm{FeCl}_{3}$ in the medium, inhibition ability of WCS358r on the pathogen was significantly reduced. It was shown that the siderophore produced WCS358r was an important factor to inhibit the growth of the mycelium and the germination of the conidia of the pathogen (Chen et al., 2006). The antagonistic experiment of Eucalyptus gray mold also showed that the siderophores of Pseudomonas was some other important factor controlling the Eucalyptus gray mold (Ran et al., 2005). Yu et al. (2017) reported that the siderophore synthesized by BAF.1 strain of Pseudomonas Syringa has strong antibacterial activity to Fusarium spp. and also has bacteriostasis to other 11 plant pathogenic fungus, which is to inhibit the germination of fungal spores and cause the change of hypha morphology (Yu et al., 2017). Sulochana et al. (2014) proved that the siderophores purified ferrite from KB culture medium of Pseudomonas aeruginosa strain JAS-25 showed strong antagonistic activity to Fusarium spp. and Aspergillus (Sulochana et al., 2014).

Brevibacillus brevis GZDF3 (CGMCC No. 10121) is an antagonistic bacteria (Zhu et al., 2017), Gram-positive bacteria, bacillus and spores separated from the rhizosphere soil of Pinellia ternata from Journal of Chinese Medicinal Materials in Guizhou province. The results showed that the strain not only had a strong antagonistic effect on the Pectobacterium carotovorum subsp. carotovorum, Fusarium oxysporum and Fusarium solani, but also a variety of pathogenic bacteria such as Fusarium pepper, Curvularia leaf spot of maize and Villosiclava virens and their antibacterial components are worth studying in depth (Shi et al., 2015). For this reason, we sequence the genome and found that there was a synthetic genetic cluster in the GZDF3 genome with $83 \%$ similarity of Bacillus anthraci Petrobactin synthesis, but no Bacillibactin synthesis gene cluster was found in the genome. Presently, no reports on the bacteriostasis of Petrobactin are reported. Therefore, this study will further use bioinformatics to analyze the gene cluster of Petrobactin synthesis of GZDF3 strain, construct the phylogenetic tree of synthetic gene, use the common CAS to detect siderophores and carry out quantitative analysis and bacteriostasis experiment, which lays the foundation for the later development and application.

\section{Materials and Methods}

\section{Experimental Materials}

Fusarium oxysporum and Brevibacillus brevis GZDF3 were isolated and preserved in the Department of Biotechnology, College of Biology and Engineering, Guizhou Medical University. Whole genome sequence of Brevibacillus brevis GZDF3 (accession number: LVYG00000000).

Genome Mining and Synthetic Gene Cluster Analysis of Siderophore Synthesis Gene of Brevibacillus brevis GZDF3

The whole genome sequence was used as material and the secondary metabolite prediction software AntiSMASH predicted the secondary metabolite synthesis gene cluster of Brevibacillus brevis GZDF3 (LVYG00000000) (Weber et al., 2015). ORFfinder and BlastP are further used to analyze gene clusters.

The local database of Brevibacillus brevis GZDF3 DNA sequence was constructed by sequence analysis software BioEdit 7.0.9.0. Taking the nucleotide sequence 
of asbABCDEF gene of Bacillus anthracis Petrobactin in NCBI as the leader sequence (GenBank: AE017334.2). Blastn was used to search the DNA sequence database of Brevibacillus brevis GZDF3 for mining the synthetic gene. AsbABCDEF used DNAMAN 4.0 for amino acid sequences alignment (Hall, 1999).

\section{Construction of Phylogenetic Tree}

Multiple sequence comparison was carried out through the BlastP online search provided by NCBI with the homologous amino acid sequence to the AsbABCFEF strain of Brevibacillus brevis GZDF3. The NeighborJoining method (NJ) was utilized to construct the phylogenetic tree of six synthetic genes (Kummar et al., 2016). Staphylococcus aureus (BAX04613.1), Streptomyces malaysiensis (ATL83423.1), Coccidioides immitis RS (XP_001245522.2), Streptomyces malaysiensis (ATL83423.1), Deinococcus maricopensis DSM 21211 (ADV68839.1), Neorhizobium galegae bv. orientalis (CDZ62393.1) and Streptomyces clavuligerus (EFG10332.1) were selected separately as outgroup. The NJ tree was constructed with the programs MEGA 7.0.14. and use the self spreading value (Bootstrap) to test its reliability. The repetition time was 1000 for assessing the evolutionary relationship between different bacteria siderophore AsbABCDEF.

Synthesis and Type Identification of Siderophore from Brevibacillus brevis GZDF3

\section{Synthesis of Siderophore}

To screen for siderophore production, GZDF3 was inoculated in modified Sugar-Aspartic acid (SA) medium $(20 \mathrm{~g} / \mathrm{L}$ sucrose, $2.0 \mathrm{~g} / \mathrm{L}$ asparagine, $0.5 \mathrm{~g} / \mathrm{L}$ $\mathrm{K}_{2} \mathrm{HPO}_{4}$ and $0.5 \mathrm{~g} / \mathrm{L} \mathrm{MgSO}_{4}$ ) and inoculation amount of $5 \%$ in a rotary shaker at $200 \mathrm{rpm}$ and $28^{\circ} \mathrm{C}$ for $48 \mathrm{~h}$. The supernatant was centrifuged at $12,000 \mathrm{r} / \mathrm{min}$ for 10 minutes and filtered with a $0.22 \mu \mathrm{m}$ microporous membrane to remove bacteria. The production of siderophore was qualitatively determined and expressed as percent siderophore (SU) by CAS liquid detection according to the method described by Schwyn and Neilands (1987) and calculations using the following formula: $\mathrm{SU}=$ $[(\mathrm{Ar}-\mathrm{As}) / \mathrm{Ar}] \times 100 \%$. Where $\mathrm{Ar}$ is the absorbance of reference and As is the absorbance of the sample at $630 \mathrm{~nm}$. (Guo et al., 2016; Schwyn and Neilands, 1987).

\section{Detection of Siderophore by Arnow Method}

At first, $1 \mathrm{~mL}$ reagent containing nitrite molybdate with $1 \mathrm{~mL}$ of $1 \mathrm{M} \mathrm{NaOH}$ was added to $1 \mathrm{~mL}$ culture filtrate and $1 \mathrm{~mL}$ of $0.5 \mathrm{M} \mathrm{HCl}$. The color change of the solution was observed. The catechol solution of $1 \mathrm{~mL}$ $100 \mu \mathrm{M}$ was used as a positive control and water as negative control (Ramasamy et al., 2016).
Effect of Culture Time on the Synthesis of Siderophore by Antagonistic Bacteria GZDF3

GZDF3 was activated to prepare bacterial culture medium. The inoculation amount of 5\% was connected to $250 \mathrm{~mL} \mathrm{SA}$ medium at $28^{\circ} \mathrm{C}$ and $200 \mathrm{r} / \mathrm{min}$ cultured for $6 \mathrm{~h}, 12 \mathrm{~h}, 18 \mathrm{~h}, 24 \mathrm{~h}, 30 \mathrm{~h}, 36 \mathrm{~h}, 42 \mathrm{~h}, 48 \mathrm{~h}, 54 \mathrm{~h}$ and $60 \mathrm{~h}$ respectively. The growth density cell was measured by the spectrophotometer at $\mathrm{OD}_{600} \mathrm{~nm}$ and CAS liquid detection method was used to initially detect the content of synthetic siderophore (Arora and Verma, 2017).

\section{The Antimicrobial Activity of Antagonistic Bacterium GZDF3 and its Synthetic Siderophore to Fusarium oxysporum}

Pathogenic fungi: Pathogenic fungi Fusarium oxysporum was isolated and preserved in the laboratory. It was inoculated in the PDA plate to activate $7 \mathrm{~d}$ and absorbed $1 \mathrm{~mL}$ aseptic water drops added to the purified fungal culture plate. The hypha was eluted and added to the $30 \mathrm{~mL}$ PDB liquid medium at $28^{\circ} \mathrm{C}$ and $200 \mathrm{r} / \mathrm{min}$ cultured for $24 \mathrm{~h}$ and then the mycelium suspension $\left(1 \times 10^{8} / \mathrm{mL}\right)$ was made with aseptic water for reserve.

Preparation of NA culture solution of antagonistic bacteria GZDF3: The seed solution was inoculated into $250 \mathrm{~mL}$ NA liquid medium $(0.3 \%$ Beef Extract, $1 \%$ peptone, $0.5 \% \mathrm{NaCl}$ ) according to the inoculation amount of $5 \%$ and then was centrifuged at $12000 \mathrm{r} / \mathrm{min}$ for $10 \mathrm{~min}$ after $48 \mathrm{~h}$ on the rocking bed of $28^{\circ} \mathrm{C}$ and 200 $\mathrm{r} / \mathrm{min}$; the supernatant was taken for reserve. Preparation of SA culture solution of antagonistic bacteria GZDF3: The seed solution was inoculated into $250 \mathrm{~mL}$ SA medium at $28^{\circ} \mathrm{C}$ and $200 \mathrm{r} / \mathrm{min}$ cultured for $48 \mathrm{~h}$ and centrifuged at $12000 \mathrm{r} / \mathrm{min}$ for $10 \mathrm{~min}$. The supernatant was filtrated by $0.22 \mu \mathrm{m}$ microporous membrane for filtration and bacteria removal and then reserve.

The agar punching method: The spore suspension was evenly coated on the PDA plate. Each plate was punched with a perforator at $2 \mathrm{~cm}$ from the center of the plate (diameter $8 \mathrm{~mm}$ ) and each plate was hit 4 holes. $150 \mu \mathrm{L}$ antagonistic NA culture and SA culture medium were added to each two holes respectively and $150 \mu \mathrm{L}$ of uninoculated NA medium and SA medium were added to the two wells on the other plate. The size of the bacteriostasis was measured after constant temperature culture at $28^{\circ} \mathrm{C}$ for $1-2 \mathrm{~d}$.

\section{Results}

The Mining of Antagonistic Bacteria GZDF3 Siderophore

In the genome of Brevibacillus brevis GZDF3, there are multiple clusters of genes encoded by different secondary metabolites, of which cluster 37 is a siderophore gene cluster as showed in (Fig. 1A). And it 
reaches $83 \%$ similarity to the biosynthetic gene cluster of Bacillus anthraci Petrobactin siderophore. Further analysis of the gene cluster by ORFfinder and BlastP revealed that the gene cluster had 15 ORF. The similarity was $54.66 \%$ between ORF8 and the synthetic gene AsbA of Bacillus anthraci Petrobactin siderophore, 43.63\% between ORF9 and AsbB, 48.17\% between ORF10 and AsbC, 51.65\% between ORF11 and AsbD, 51.06\% between ORF 12 and AsbE and 52.14\% between ORF13 and AsbE. The synthetic gene asbABCDEF of Bacillus anthraci Petrobactin siderophore was used for the local Blast comparison with Brevibacillus brevis GZDF3 and it was found that it was corresponding to GENE5689, GENE5690, GENE5691, GENE5692, GENE5693 and GENE5694 of Brevibacillus brevis GZDF3 respectively as shown in (Fig. 1B).

\section{Construction of Phylogenetic Tree}

The NJ tree of AsbABCDEF is constructed by MEGA 7.0.14 software (Fig. 2). From the whole, the siderophore AsbABCDEF with different bacteria can be divided into two groups that the Brevibacillus brevis GZDF3 and the bacillus of short bacillus are clustered into a class and the Bacillus anthracis, Bacillus thuringiensis and Bacillus cereu are clustered into a class. AsbA, AsbB, AsbD and AsbE of Brevibacillus brevis GZDF3 were all clustered into a small branch with Brevibacillus brevis NBRC 100599, while AsbC was isolated to one branch; AsbF and Brevibacillus brevis ATCC 35690 were clustered into a branch. While on the other group, four synthetase genes of AsbABCD were all that Bacillus anthraci and Bacillus thuringiensis clustered into one branch and Bacillus cereus was isolated to the other branch. AsbE was that Bacillus anthraci and Bacillus cereus clustered into a branch and Bacillus thuringiensis was isolated to a branch. AsbF was that Bacillus cereus and Bacillus thuringiensis were clustered into a branch and Bacillus anthracis was isolated to a branch.

\section{Synthesis and Identification of Siderophore}

GZDF3 was cultured on the SA medium for $48 \mathrm{~h}$, as depicted in (Fig. 3A), GZDF3 produced siderophores that are represented by dark red after specific color reaction of CAS detection solution. According to the formula $\mathrm{SU}=[(\mathrm{Ar}-\mathrm{As}) / \mathrm{Ar}] \times 100 \%$, the maximum siderophore production was obtained $(\mathrm{SU}=27.035 \%)$ after $48 \mathrm{~h}$ of incubation, which indicated that Brevibacillus brevis GZDF3 can synthesize siderophore. And using the Arnow's assay, we observed the formation of a red-orange color (Fig. 3B), which indicated that GZDF3 produced the catecholates species siderophore.

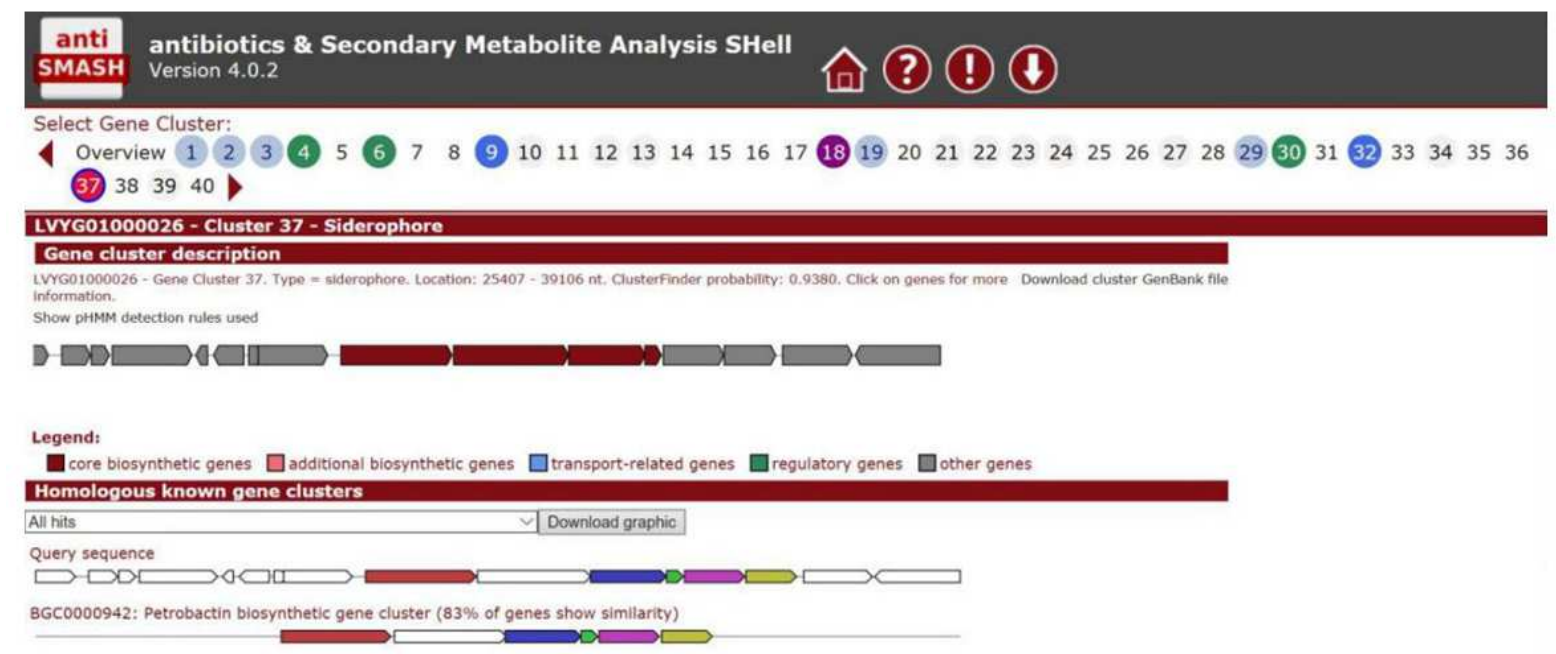

(A)

AsbA AsbB AsbC AsbD AsbE AsbF

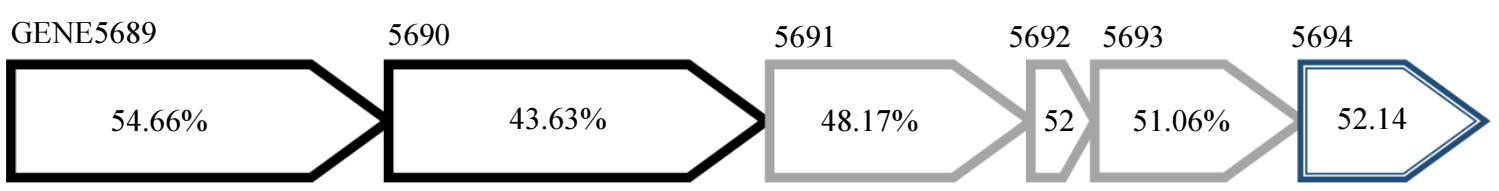

(B)

Fig. 1: (A) Analysis on the secondary metabolites of Brevibacillus brevis GZDF3 strain genome; (B) Target gene and synthetic gene of Brevibacillus brevis Petrobactin siderophores 


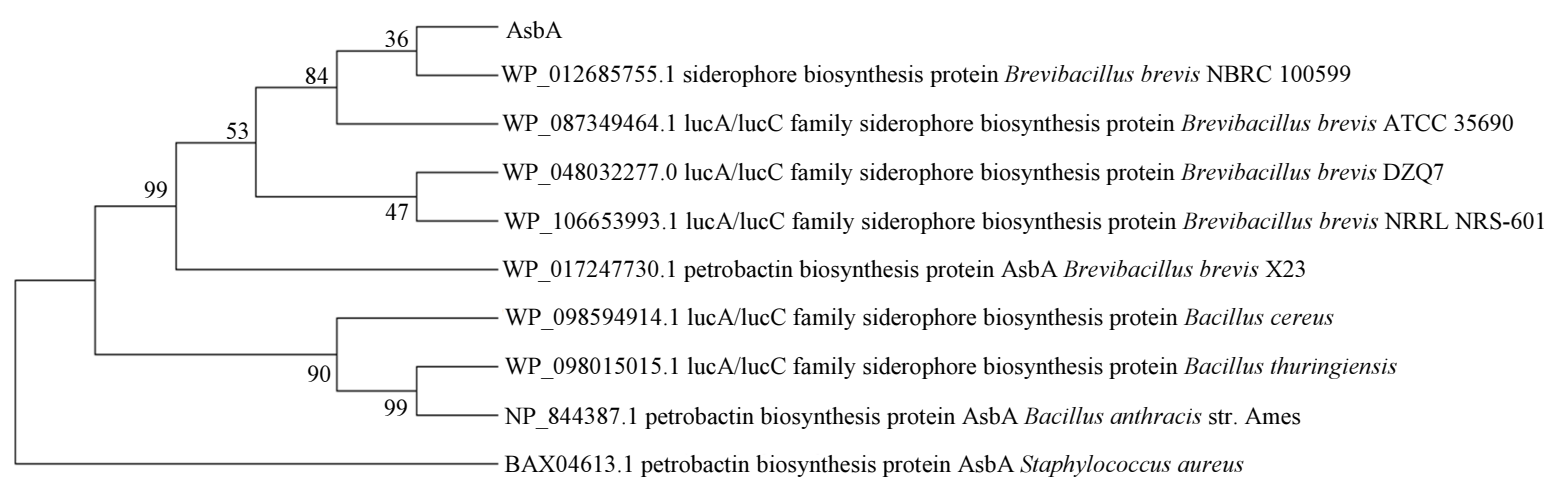

(A)

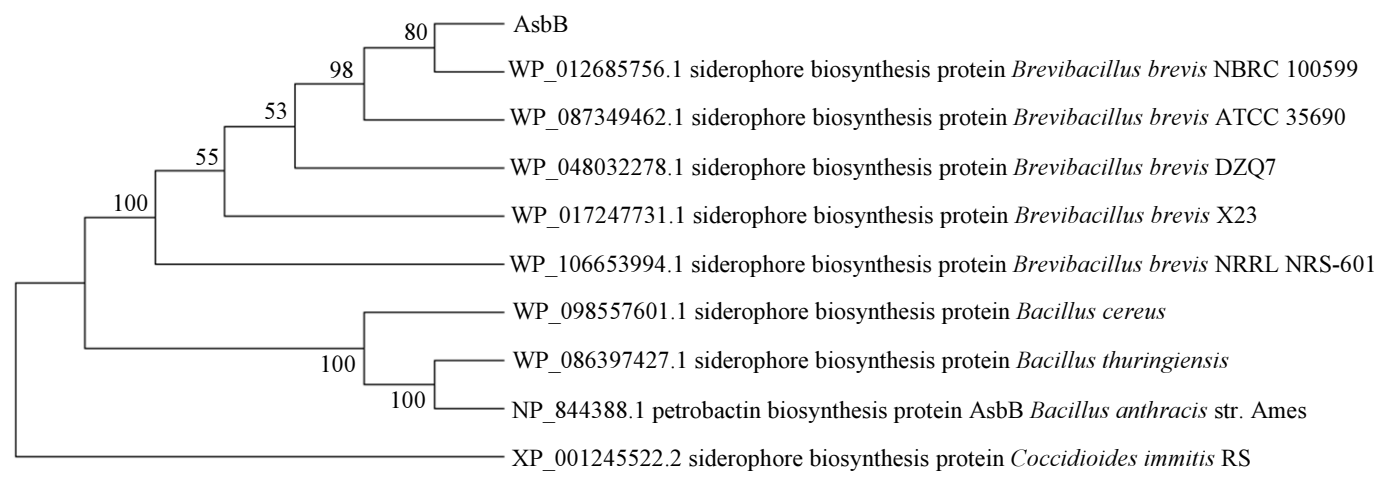

(B)

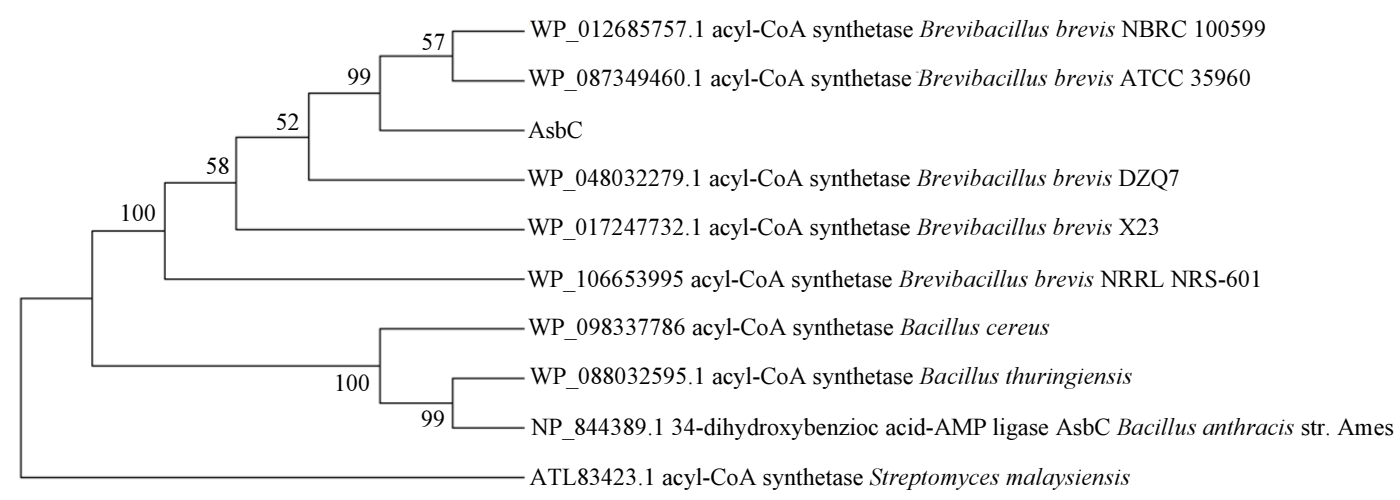

(C)

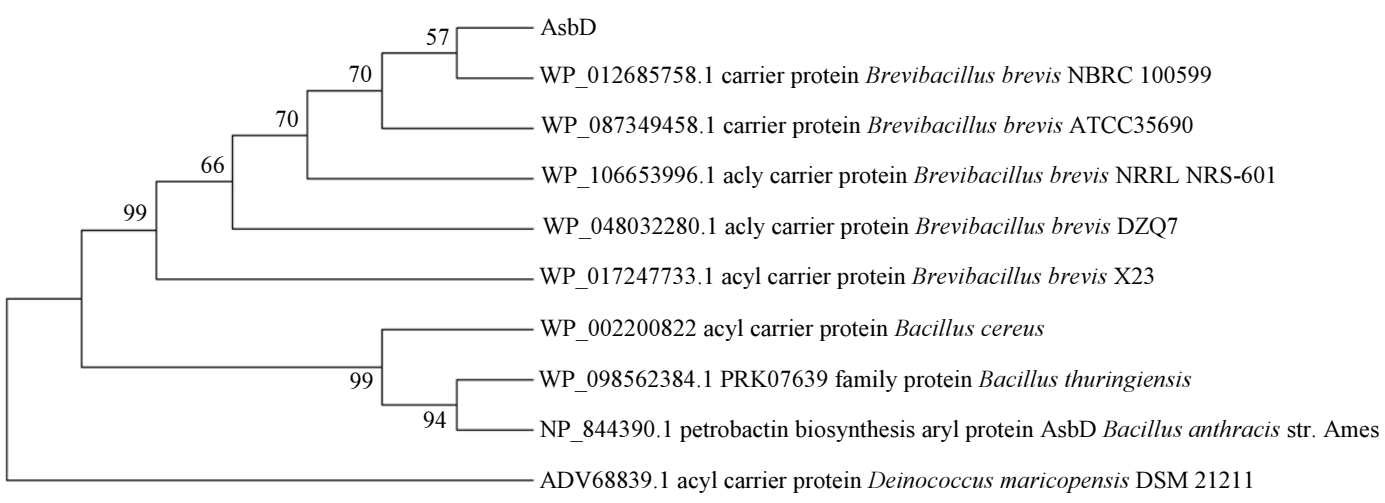

(D) 


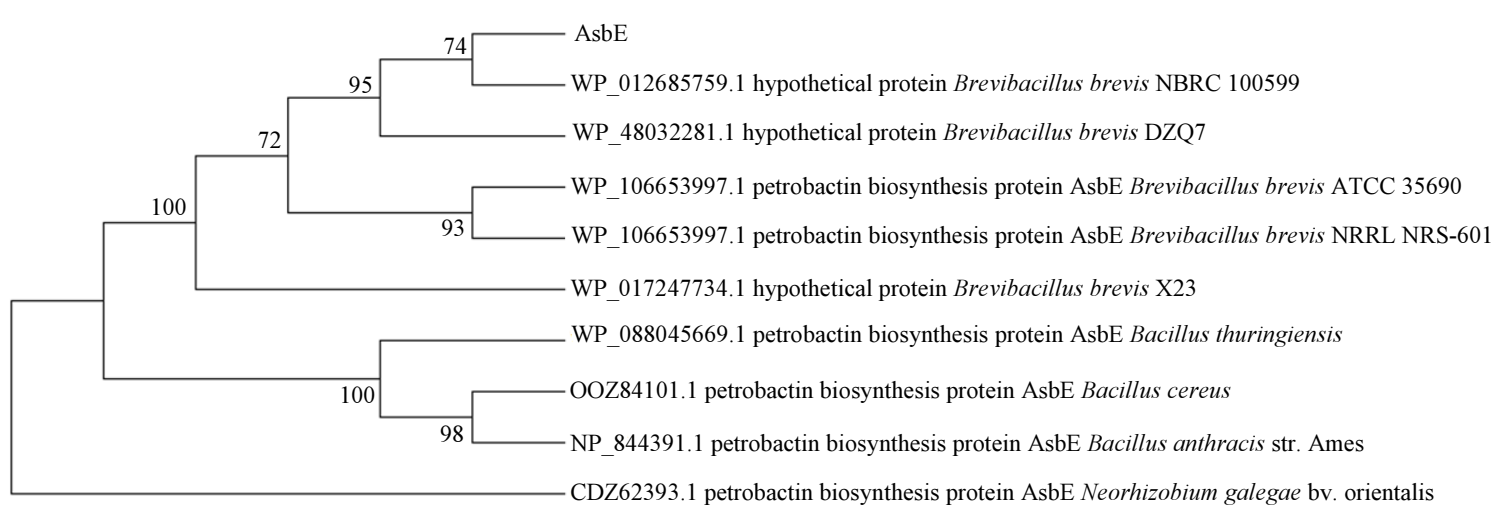

(E)

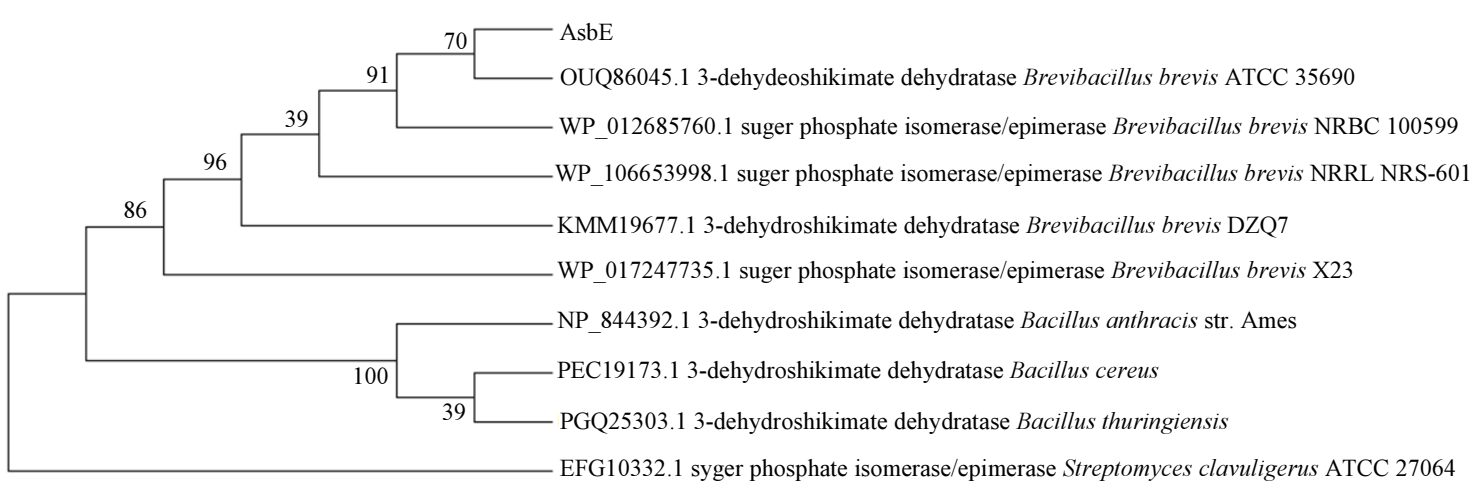

$(\mathrm{F})$

Fig. 2: (A) The Phylogenetic tree of siderophore synthetase AsbA; (B) The Phylogenetic tree of siderophore synthetase AsbB; (C) The Phylogenetic tree of siderophore synthetase AsbC; (D) The Phylogenetic tree of siderophore synthetase AsbD; (E) The Phylogenetic tree of siderophore synthetase AsbE; (F) The Phylogenetic tree of siderophore synthetase AsbF
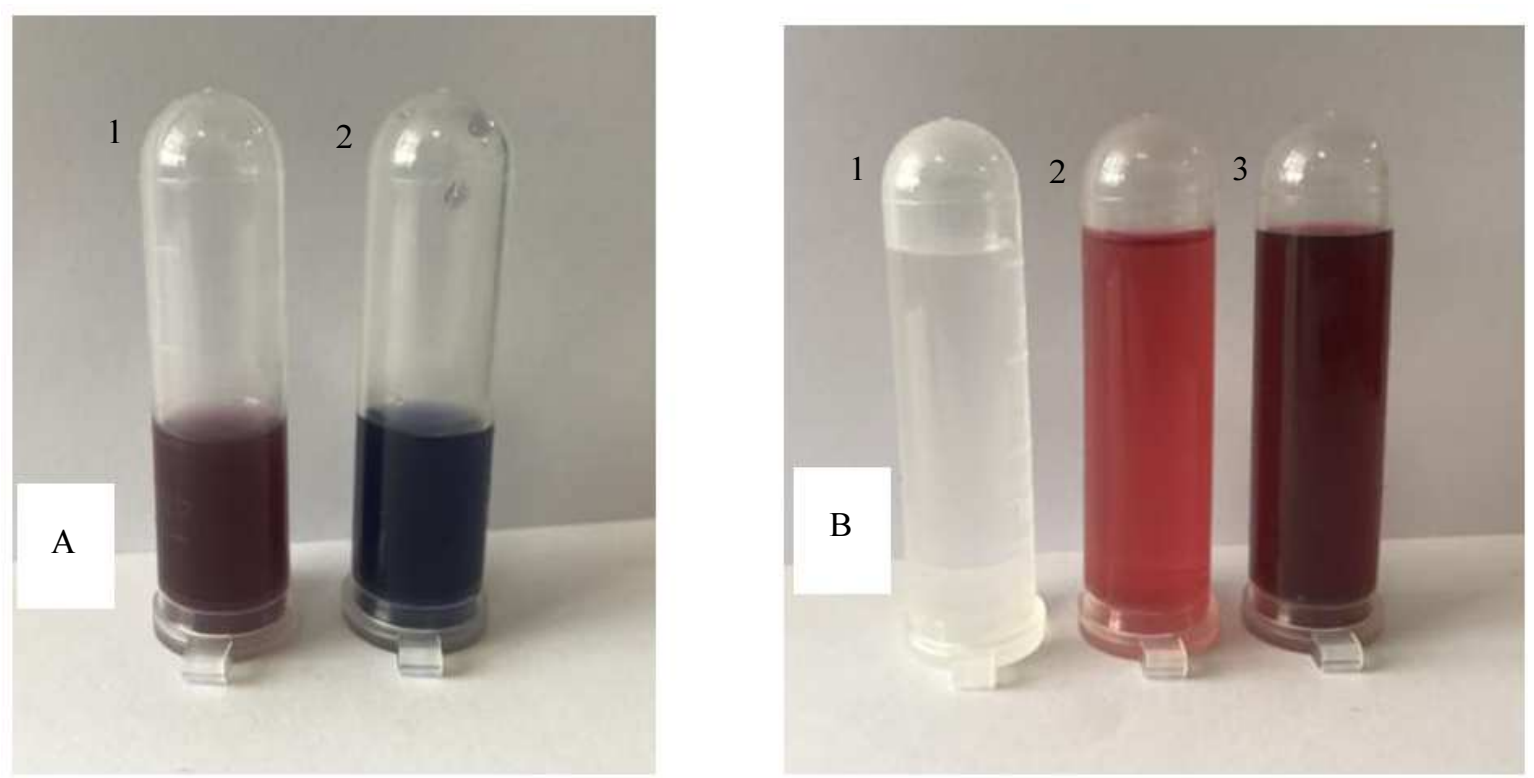

Fig. 3: Synthesis and type detection of siderophore; (A) CAS liquid detection: 1. Iron free fermentation liquid supernatant; 2. Negative; (B) Arnow experiment: 1. Negative; 2. Iron free fermentation liquid supernatant; 3. Catechol solution 


\section{Optimum Culture Time for the Synthesis} Siderophore of Bacillus Subtilis GZDF3

As shown in (Fig. 4), the synthesis of GZDF3 siderophore in the SA medium was affected by the incubation time and it reached two peaks when the incubation time was $30 \mathrm{~h}$ and $48 \mathrm{~h}$. Meanwhile, the activity unit $\mathrm{SU}$ of the siderophore was $24.09 \%$ and $24.20 \%$ at $\mathrm{OD}_{630} \mathrm{~nm}$ respectively and the synthesis of siderophore was not detected during $0-18 \mathrm{~h}$. And the growth curve of Brevibacillus brevis GZDF3 cultured in SA medium showed that it was basically consistent with the four growth stages of bacteria at $\mathrm{OD}_{600} \mathrm{~nm}$.

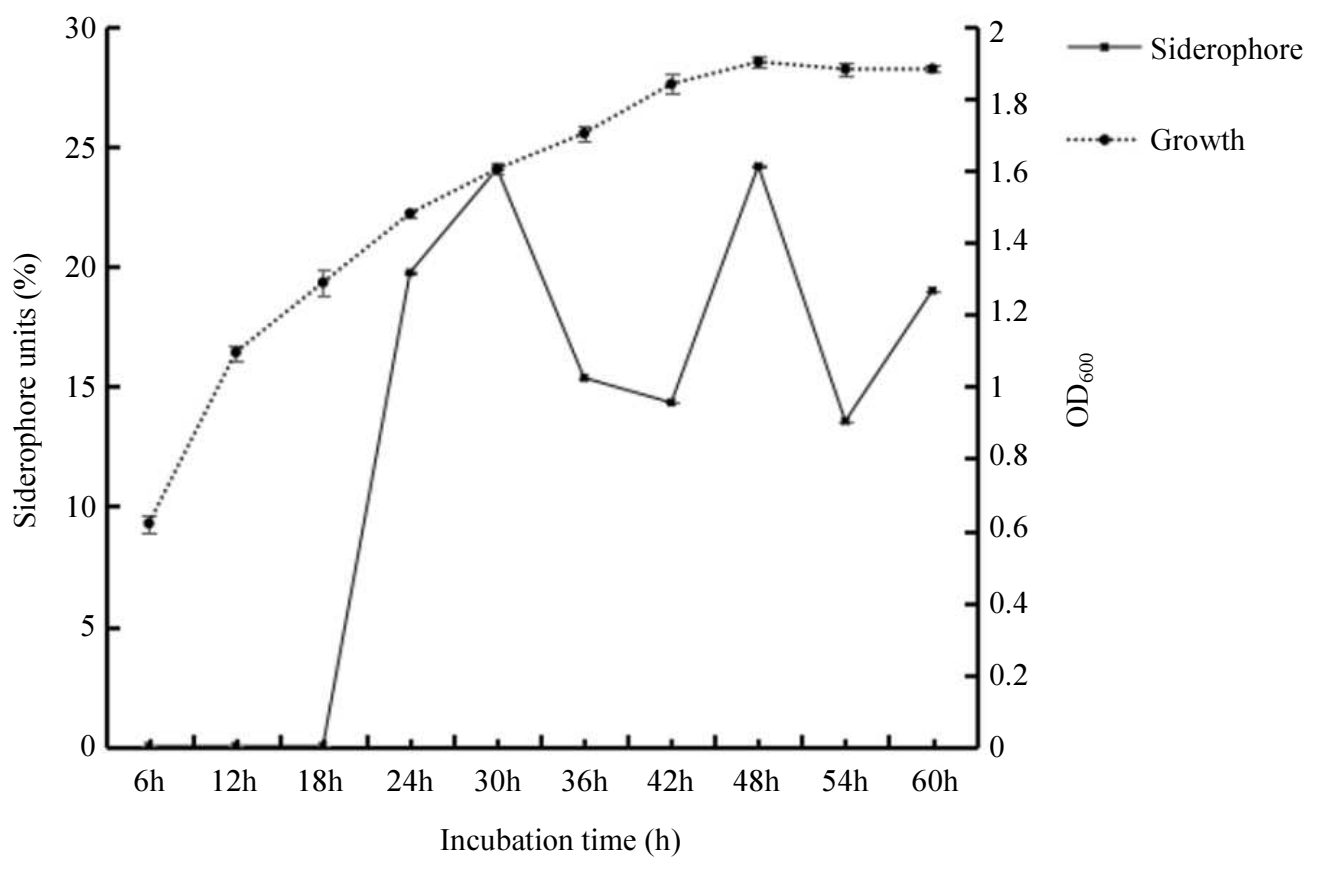

Fig. 4: Variation of siderophore production and cell growth with different incubation time; Error bars represent standard deviations of three replicates
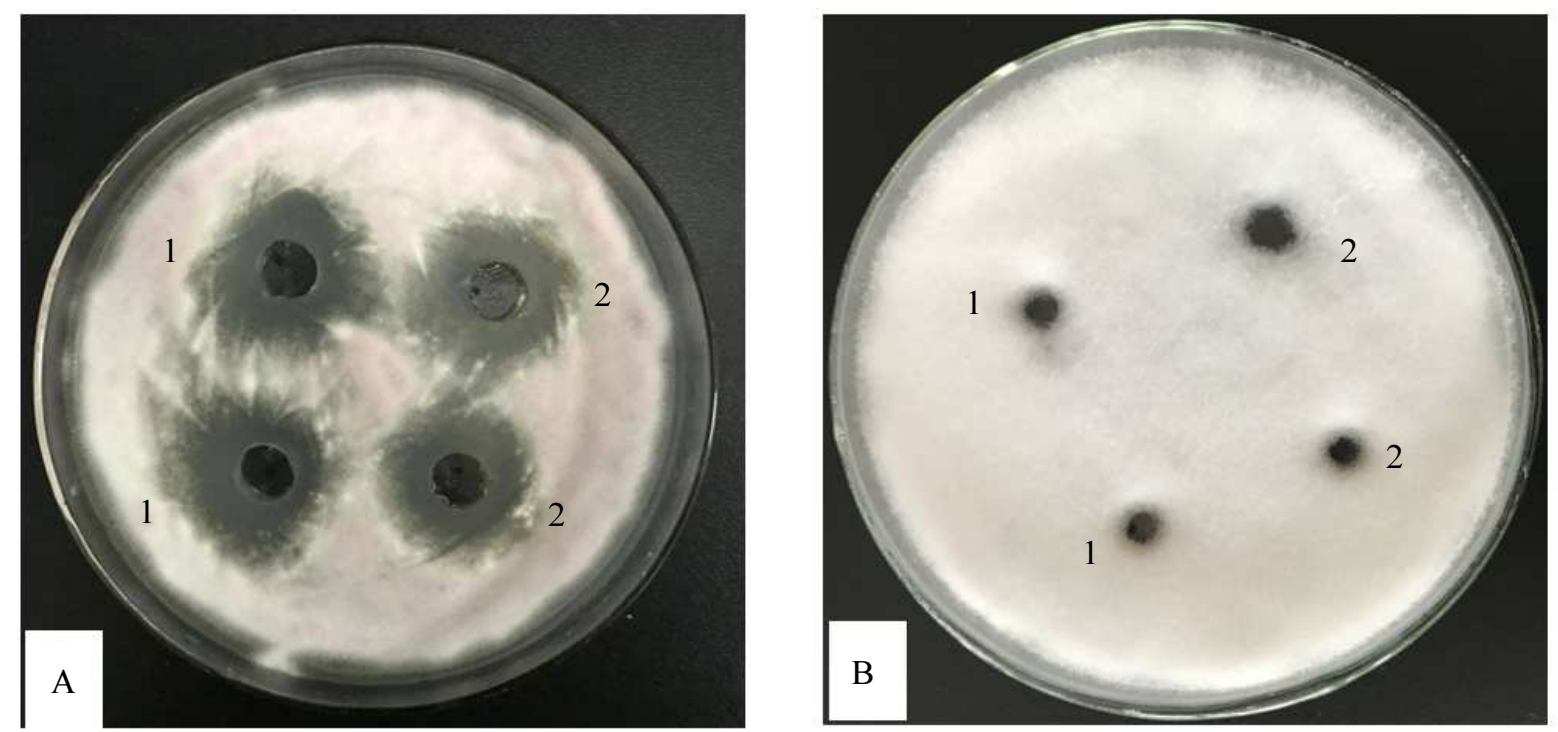

Fig. 5: The antagonistica activity of Brevibacillus brevis GZDF3 (A) Fermentation liquid medium: 1. antagonistic NA culture; 2. antagonistic NA culture; (B) Uninoculated liquid medium: 1. NA culture; 2. SA culture 
Bacteriostatic Activity of the Siderophore Synthesized by Bacillus Subtilis GZDF3 on Fusarium Oxysporum

As shown in (Fig. 5A1), the GZDF3 has a strong antagonism to Fusarium oxysporum that was one of the Pathogenic fungus causing rotted root on Pinellia ternata and the inhibition zone was $18-20 \mathrm{~mm}$; while the bacteriostasis of the siderophore fermented and synthesized by iron free medium on Fusarium oxysporum was shown in (Fig. 5A2) and the inhibition zone was 15-16 mm. As shown in (Fig. 5B), uninoculated NA and SA liquid medium were none inhibition zone. The results showed that siderophore had obvious bacteriostasis on Fusarium oxysporum of Pathogenic bacteria of rotted root on Pinellia ternata.

\section{Conclusion}

Petrobactin siderophore was first found in Marinobacter hydrocarbonoclasticus (Barbeau et al., 2002). It was further found that the Bacillus anthraci strains and Bacillus cereus strains can also synthesize Petrobactin (Wilson et al., 2006). For Bacillus anthracis, it can synthesize Petrobactin and Bacillibactin. Petrobactin is the main siderophore in Bacillus anthracis. Because it is not identified by the innate immune system and it has been found that the Petrobactin is the cardinal factor in the anthrax model, most of which is the mixed type of catechol-carboxylate siderophore (Nusca et al., 2012). It appears that Bacillibactin has higher importance in Bacillus cereus on insect model experiments (Hayrapetyan et al., 2016). In this study, a siderophore synthesis gene cluster with Petrobactin similarity of $83 \%$ was discovered, while no Bacillibactin siderophore was found. At present, functions of Petrobactin and Bacillibactin synthetic genes as $B A B C D E F$ and $d h b B C F$ have been researched and reported (Segond et al., 2014). Cendrowski et al. (2004) reviewed the functions of gene $a s b A$ by directional gene knockout. Under the condition of iron deficiency, the asbA deletion mutant showed a decrease in the siderophore yield and a weaker growth. Moreover, the pathogenicity of as $b A$ deletion mutant mice was also significantly reduced (Cendrowski et al., 2004). Nusca et al. (2012) realized the heterologous expression of Petrobactin siderophore by using citric acid, spermidine and 3, 4-two hydroxybenzoic acid substrates. It is found that the function of AsbA synthase can be compensated by AsbB. The strain deletion of AsbA synthetase with the other five genes can also synthesize part of Petrobactin (Nusca et al., 2012). The asbE gene deletion cannot synthesize Petrobactin siderophore showed that $a s b E$ gene was also a key gene in the pathway of Petrobactin siderophore synthesis. The function and structure of asbB (Nusca et al., 2012), asbD (Schmelz et al., 2009) and $a s b F$ (Pfleger et al., 2008) in Petrobactin siderophore synthesis gene cluster have been investigated. Domagalski et al. (2013) determined the crystal structure of different chorismic acid synthetase DhbC of Bacillibactin siderophore in Bacillus anthraci. DhbC participated in the biosynthesis of 2, 3-two hydroxybenzoate (DHB) and methyl naphthoquinone (MK) of the respiratory chain group; the consumption of DhbC led to the deficiency of DHB, which showed the importance of $\mathrm{DhbC}$ in siderophore biosynthesis (Domagalski et al., 2013). Hertlein et al. (2014) studied the deletion of DhbF by gene inactivation mutagenesis and confirmed that $d h b$ gene cluster was in charge of the synthesis of Bacillibactin siderophore (Hertlein et al., 2014). The study predicted that Brevibacillus brevis GZDF3 can synthesize Petrobactin siderophores. Comparison analysis showed that the similarity of GZDF3 strains and Bacillus anthraci Petrobactin siderophore synthetase AsbA, AsbB, AsbC, AsbD, AsbE, AsbF were 54.66\%, 43.63\%, 48.17\%, 51.65\%, 51.06\%, $52.14 \%$ respectively. The phylogenetic tree of six siderophores synthesis AsbABCDEF showed that the siderophore synthetase was mainly divided into two groups and the siderophore synthetase of Brevibacillus brevis genus was clustered into one branch and the other bacterial sources were gathered into the other branch. Among the different bacterial sources, except that AsbF synthetase was Bacillus anthraci clusters alone, AsbE synthase was that Bacillus anthraci and Bacillus cereus clustered into a branch, the other four synthases was all that Bacillus anthraci and Bacillus thuringiensis clustered into a branch. It is identified by CAS liquid detection and Arnow's assay that the siderophore synthesized by Brevibacillus brevis GZDF3 was catecholates but the type synthesized by Bacillus anthraci was a mixture of catechol-carboxylate.

Siderophore in biocontrol bacteria has strong antagonism against various pathogenic fungus, while the inhibition on Petrobactin siderophore is not yet available. Li et al. (2014) found that Bacillus amylus SQR9 has a broad-spectrum antifungal activity. When six fungal pathogens such as Fusarium oxysporum and Rhizoctonia solani were found, five synthetic genes of Bacillibactin siderophores in the strains all express up-regulation. While in the mutant strains with deficiency of Bacillibactin synthetic gene, the antifungal activity decreased or even no antibacterial activity. It indicates that Bacillibactin siderophore plays a major role in the control of fungal diseases (Li et al., 2014). Bacillus anthracis Petrobactin siderophore is closely related to the virulence of Bacillus anthracis (Cendrowski et al., 2004) and the Petrobactin siderophore is a NRPS-NIS mixed. However, there is no report on the bacteriostasis of Petrobactin siderophore in the biocontrol bacteria. In this study, the GZDF3 strain used iron free medium to 
promote the synthesis of siderophores and its supernatant showed strong antagonistic activity to Fusarium oxysporum. Based on this, we believe that the synthesis regulation and bacteriostasis activity of Brevibacillus brevis GZDF3 siderophore are worthy of further studys.

This study preliminaries revealed that the mining and identification of Brevibacillus brevis GZDF3 to Petrobactin siderophore and the strong bacteriostasis to Pathogenic fungi Fusarium oxysporum, which provides the basis for further separation and purification and functional verification, especially in the prevention and control of fungi in Guizhou medicinal herbs. Meanwhile, it has great significance to the protection and further development and application of traditional Chinese medicinal materials.

\section{Acknowledgement}

This study was supported by the project ([2013]5014) of the Modernization of Traditional Chinese Medicine of Guizhou, the project (2016002) of Medical Biotechnology Engineering Research Center, Guizhou Medical University and the project ([2017]5-33) of the United Fund Project of Guiyang.

\section{Author's Contributions}

Miaomiao Sheng and Huake Jia: Contributed to the planning and implementation of this study as well as interpretation of article preparation and drafted the manuscript.

Xiaomai Tao, Lina Zeng, Tingting Zhang, Zuquan Hu and Zhu Zeng: Coordinated the data-analysis and assisted in the writing of the manuscript.

Hongmei Liu: Contributed to the planning and implementation of research work and revising the article.

\section{Conflict of Interest}

The authors declare that they have no competing interests. The corresponding author affirms that all of the authors have read and approved the manuscript.

\section{References}

Arora, N.K. and M. Verma, 2017. Modified microplate method for rapid and efficient estimation of siderophore produced by bacteria. Biotech, 7: 381-381. DOI: $10.1007 / \mathrm{s} 13205-017-1008-y$

Barry, S.M. and G.L. Challis, 2009. Recent advances in side rophore biosynthesis. Curr. Opin Chem. Biol., 13: 205-215. DOI: 10.1016/j.cbpa.2009.03.008

Barbeau, K., G. Zhang, D.H. Live and A. Butler, 2002. Petrobactin, a photoreactive siderophore produced by the oil-degrading marine bacterium Marinobacter hydrocarbonoclasticus. J. Am. Chem. Soc., 124: 378-379. PMID: 11792199
Carroll, C.S., C.L. Grieve, I. Murugathasan, A.J. Bennet and C.M. Czekster et al., 2017. The rhizoferrin biosynthetic gene in the fungal pathogen Rhizopus delemar is a novel member of the NIS gene family. Int. J. Biochem. Cell Biol., 89: 136-146.

DOI: 10.1016/j.biocel.2017.06.005

Cendrowski, S., W. MacArthur and P. Hanna, 2004. Bacillus anthracis requires siderophore biosynthesis for growth in macrophages and mouse virulence. Mol. Microbiol., 51: 407-417. DOI: 10.1046/j.1365-2958.2003.03861.x

Chen, X., F.X. Cao, Z.N. Li, T.H. Wu and L.X. Ran, 2006. Role of siderophore, DAPG and PCA in suppression of conidia in Cryphonectria parasitica. Hebei J. Forestry Orchard Res., 21: 404-208.

Domagalski, M.J., K.L. Tkaczuk, M. Chruszcz, T. Skarina and O. Onopriyenko et al., 2013. Structure of isochorismate synthase $\mathrm{DhbC}$ from Bacillus anthracis. Acta Crystallogr Sect. F Struct. Biol. Cryst. Commun., 69: 956-61.

DOI: $10.1107 / \mathrm{S} 1744309113021246$

Fukushima, T., B.E. Allred, A.K. Sia, R. Nichiporuk and U.N. Andersen et al., 2013. Grampositive siderophore-shuttle with ironexchange from Fe-siderophore to aposiderophoreby Bacillus cereus YxeB. Proc. Natl. Acad. Sci. USA., 110: 13821-13826. DOI: 10.1073 /pnas. 1304235110

Grossman, T.H., M. Tuckman, S. Ellestad and M.S. Osburne, 1993. Isolation and characterization of Bacillus subtilis Genes Involved in Siderophore Biosynthesis: Relationship between B. subtilis sfpo and Escherichia coli entD Genes. J. Bacteriol., 175: 6203-6211.

Guo, H., Y. Yang, K. Liu, W. Xu and J. Gao et al., 2016. Comparative genomic analysis of Delftiatsuruhatensis MTQ3 and the identification of functional NRPS genes for siderophore production. BioMed. Res. Int., 2016: 3687619-3687619. DOI: $10.1155 / 2016 / 3687619$

Hagan, A.K., P.E. Carlson and P.C. Hanna, 2016. Flying under the radar: The non-canonical biochemistry and molecular biology of petrobactin from Bacillus anthracis. Mol. Microbiol., 102: 196-206. DOI: 10.1111/mmi. 13465

Hall, T.A., 1999. BioEdit: A user-friendly biological sequence alignment editor and analysis program for Windows 95/98/NT. Nucl. Acids Symp. Ser., 41: 95-98.

Han, A.W., M. Sandy, B. Fishman, A.E. Trindade-Silva and C.A. Soares et al., 2013. Turnerbactin, a novel triscatecholate siderophore from the shipworm endosymbiont Teredinibacter turnerae T7901. PLoS One, 8: e76151-e76151. DOI: 10.1371/journal.pone.0076151 
Hayrapetyan, H., R. Siezen, T. Abee and M. Groot. Nierop, 2016. Comparative genomics of irontransporting systems in Bacillus cereus strains and impact of iron sources on growth and biofilm formation. Front Microbiol., 7: 842-842. DOI: $10.3389 /$ fmicb.2016.00842

Hertlein, G., S. Muller, E. Garcia-Gonzalez, L. Poppinga and R.D. Sussmuth et al., 2014. Production of the catechol type siderophore bacillibactin by the honey bee pathogen paenibacillus larvae. PLoS One, 9: e108272-e108272.

DOI: 10.1371/journal.pone.0108272

Hollensteiner, J., F. Wemheuer, R. Harting, A.M. Kolarzyk and S.M. Diaz Valerio et al., 2016. Bacillus thuringiensis and Bacillus weihenstephanensis Inhibit the Growth of Phytopathogenic Verticillium Species. Front Microbiol., 7: 2171-2171.

DOI: $10.3389 /$ fmicb.2016.02171

Kummar, S., G. Stecher and K. Tamura, 2016. MEGA7: Molecular evolutionary genetics analysis version 7.0 for bigger datasets. Mol. Biol. Evol., 33: 1870-1874. DOI: 10.1093/molbev/msw054

Li, B., Q. Li, Z. Xu, N. Zhang and Q. Shen et al., 2014. Responses of beneficial Bacillus amyloliquefaciens SQR9 to different soilborne fungal pathogens through the alteration of antifungal compounds production. Front Microbiol., 5: 636-636.

DOI: $10.3389 /$ fmicb.2014.00636

Lee, J.Y., B.K. Janes, K.D. Passalacqua, B.F. Pfleger and N.K. Bergman et al., 2007. Biosynthetic analysis of the petrobactin siderophore pathway from Bacillus anthracis. J. Bacteriol., 189: 1689-710.

DOI: 10.1128/JB.01526-06

Nusca, T.D., Y. Kim, N. Maltseva, J.Y. Lee and W. Eschenfeldt et al., 2012. Functional and structural analysis of the siderophore synthetase $A s b B$ through reconstitution of the petrobactin biosynthetic pathway from Bacillus anthracis. J. Biol. Chem., 287: 16058-16072. DOI: 10.1074/jbc.M112.359349

Pfleger, B.F., Y. Kim, T.D. Nusca, N. Maltseva and J.Y. Lee et al., 2008. Structural and functional analysis of $A s b F$ : origin of the stealth 3,4-dihydroxybenzoic acid subunit for petrobactin biosynthesis. Proc. Natl. Acad. Sci. USA., 105: 17133-17138. DOI: $10.1073 /$ pnas.0808118105

Ran, L.X., M.L. Xiang, B. Zhou and A. Peter, 2005. Siderophore are the main determinats of fluorescent Pseudomonas strains in suppression of grey mould in Eucalyptus urophylla. Acta Phytopathol. Sinica, 35: 6-12.

Ramasamy, A., S. Muthusamy, A. Arthanari, R.M. Joseph and V. Govindasami, 2016. Production of Non-Ribosomal Peptide Synthetase (NRPS)dependent siderophore by Aeromonas isolates. Iran Biomed. J., 20: 235-240.

Serrano, D.O., 2017. Biotechnology of siderophores in high-impact scientific fields. Biomol. Concepts, 8: 169-178. DOI: 10.1515/bmc-2017-0016
Schmelz, S., N. Kadi, S.A. McMahon, L. Song and D. Oves-Costales et al., 2009. AcsD catalyzes enantioselective citrate desymmetrization in siderophore biosynthesis. Nat. Chem. Biol., 5: 174-182. DOI: $10.1038 /$ nchembio. 145

Schwyn, B. and J.B. Neilands, 1987. Universal chemical assay for the detection and determination of siderophore. Anal. Biochem., 160: 47-56.

Segond, D., E. Abi Khalil, C. Buission, N. Daou and M. Kallassy et al., 2014. Iron acquisition in Bacillus cereus: The roles of IlsA and bacillibactin in exogenous ferritin iron mobilization. PLoS Pathog., 10: e1003935-e1003935.

DOI: $10.1371 /$ journal.ppat.1003935

Shi, J.L., Y.Q. Li, K.M. Hu, J.G. Ren and H.M. Liu, 2015. Isolation and identification of pathogens from rotted root of Pinelliaternatain Guizhou Province. Microbiol. China, 42: 289-299.

Storey, E.P., R. Boghozian, J.L. Little, D.W. Lowman and R. Chakraborty et al., 2006. Characterization of 'Schizokinen'; a dihydroxamate-type siderophore produced by Rhizobium leguminosarum IARI 917. Biometals, 19: 637-49. DOI: $10.1007 / \mathrm{s} 10534-006-9001-7$

Sulochana, M.B., S.Y. Jayachandra, S.K. Kumar and A. Dayanand, 2014. Antifungal attributes ofsiderophore produced by the Pseudomonas aeruginosa JAS-25. J. Basic Microbiol., 54: 418-24. DOI: $10.1002 /$ jobm.201200770

Weber, T., K. Blin, S. Duddela, D. Krug and H.U. Kim et al., 2015. antiSMASH 3.0-a comprehensive resource for the genome mining of biosynthetic gene clusters. Nucleic Acids Res., 43: W237-W243. DOI: $10.1093 /$ nar/gkv437

Wilson, M.K., R.J. Abergel, K.N. Raymond, J.E. Arceneaux and B.R. Byers, 2006. Siderophores of Bacillus anthracis, Bacillus cereus and Bacillus thuringiensis. Biochem. Biophys. Res. Commun., 348: 320-325. DOI: 10.1016/j.bbrc.2006.07.055

Yu, S., C. Teng, J. Liang, T. Song and L. Dong et al., 2017. Characterization of siderophore produced by Pseudomonas syringae BAF.1 and its inhibitory effects on spore germination and mycelium morphology of Fusarium oxysporum. J. Microbiol., 55: 877-884. DOI: 10.1007/s 12275-017-7191-z

Zawadzka, A.M., Y. Kim, N. Maltseva, R. Nichiporuk and Y. Fan et al., 2009. Characterization of a Bacillus subtilis transporter for petrobactin, an anthrax stealth siderophore. Proc. Natl. Acad. Sci. USA., 106: 21854-21859.

DOI: $10.1073 /$ pnas.0904793106

Zhu, X.H., Y.Q. Li, X.M. Tao, J.G. Ren and H.M. Liu et al., 2017. Screening and identification of antagonistic bacteria against soft rot of Pinelliaternata in Guizhou Province. Microbiol. China, 44: 438-448. 